\title{
Postpartum Family Planning Use and Its Determinants among Women of the Reproductive Age Group in Low-Income Countries of Sub-Saharan Africa: A Systematic Review and Meta- Analysis
}

\author{
Tesfalem Tilahun Yemane $\mathbb{D}^{1},{ }^{1}$ Getahun Gebre Bogale $\mathbb{D}^{\mathrm{D}},{ }^{2}$ Gudina Egata $\mathbb{D}^{3},{ }^{3}$ \\ and Tilahun Kassa Tefera ${ }^{1}{ }^{1}$ \\ ${ }^{1}$ Department of Nursing, Dessie Health Science College, Dessie, Ethiopia \\ ${ }^{2}$ Department of Health Informatics, School of Public Health, College of Medicine and Health Sciences, Wollo University, \\ Dessie, Ethiopia \\ ${ }^{3}$ Department of Public Health Nutrition \& Dietetics, School of Public Health, College of Health Sciences, \\ Addis Ababa University, Ethiopia
}

Correspondence should be addressed to Tesfalem Tilahun Yemane; tesfalemtilahun1@gmail.com

Received 8 January 2021; Accepted 30 July 2021; Published 21 August 2021

Academic Editor: Vikas Roy

Copyright (c) 2021 Tesfalem Tilahun Yemane et al. This is an open access article distributed under the Creative Commons Attribution License, which permits unrestricted use, distribution, and reproduction in any medium, provided the original work is properly cited.

Background. Postpartum family planning is the initiation and use of family planning services within the first 12 months following childbirth. Postpartum contraceptives reduce maternal and infant mortality by preventing unplanned and unwanted pregnancies and by spacing pregnancies at least two years after the previous birth. Thus, it is usually designed as an integral part of reproductive and maternal and child health programs. Therefore, the aim of this systematic review and meta-analysis is to estimate the pooled prevalence of postpartum modern contraceptive use and identify its determinants in low-income countries of sub-Saharan Africa. Methods. A systematic review and meta-analysis of published and unpublished studies were used. PubMed, HINARI, ScienceDirect, Cochrane Library, Wiley Library, ETH Library, and Google Scholar were used to search all articles. STATA 14 software was used for data analysis. Funnel plots and Egger's test were used to examine the risk of publication bias. Heterogeneity was checked by using Cochran's $Q$ test and $I^{2}$ test. A random effect model was computed to estimate the pooled prevalence. Results. A total of 33 articles were included. The pooled prevalence of postpartum contraceptive use in low-income countries of sub-Saharan Africa was 37.41\%, 95\% CI: (31.35, 43.48\%). Secondary and above level of education (AOR 2.09, 95\% CI: $(1.52,2.86)$ ), discussion with husband (AOR 3.68, 95\% CI: $(1.96,6.89)$ ), resumption of menses (AOR: 3.98, 95\% CI: $(2.62,6.03)$ ), ANC follow-up (AOR; 5.10, 95\% CI: (3.57, 7.29)), knowledge of modern family planning (AOR: 5.65, 95\% CI: 3.58, 8.93)), and family planning counseling during ANC $(\mathrm{AOR}=5.92,95 \% \mathrm{CI}:(2.54,13.79))$ were found to be determinants of postpartum contraceptive utilization. Conclusion. In this systematic review and meta-analysis, the prevalence of postpartum modern contraceptive use was found to be low compared to the existing global recommendations. Therefore, empowering maternal education, delivering adequate counseling, and strengthening existing integrated maternal and child health services are highly recommended to increase postpartum contraceptive use. This trial is registered with CRD42020160612.

\section{Introduction}

Postpartum family planning (PPFP) is the initiation and use of family planning services within the first 12 months after childbirth to prevent unintended and closely spaced pregnancies $[1,2]$. The postpartum period is critical for addressing high unmet needs in family planning and is used for reducing the risk of closely spaced pregnancies [3]. 
According to the World Health Organization (WHO), it recommends that interpregnancy intervals should be at least 2 years [4]. Short birth intervals increase the risk for the health of both the mother and the child, such as risk of preterm birth, low birth weight and small for gestational age, increased chances of chronic undernourishment, stunted growth, and child mortality $[5,6]$. Using family planning during the postpartum period may help women to space births by at least 24 months, and this can also help to reduce maternal and child mortalities by $30 \%$ and $10 \%$, respectively [7]. When a pregnancy occurs less than six months after a previous delivery, the risk of low birth weight and prematurity doubles, and children born less than 24 months after a previous birth are $60 \%$ more likely to die during infancy than those born more than 24 months [8].

Postpartum contraceptive utilization remains low in subSaharan Africa [9]. Since the uptake of PPFP was low, the significant factors influencing the uptake of PPFP are the level of education, perinatal family planning, counselling, menses return, breastfeeding status, return of sexual activity, fear of side effects, and low perceived risk of getting pregnant [10].

Maternal health remains a major global concern since pregnancy and childbirth are the leading causes of morbidity, mortality, and disability among women of the reproductive age group [11]. Globally, more than 9 out of 10 women want to avoid pregnancy for 2 years after childbirth [2]. According to DHS data from 21 low- and middle-income countries between 2005 and 2012, almost all (95\%) women 0-12 months postpartum wanted to avoid pregnancy in the next 24 months, but less than one-third (31\%) were using any method of contraception. Sixty-one percent (61\%) of postpartum women had an unmet need for family planning [3, 12].

According to studies done in five low-income countries, the rates of postpartum modern contraceptive usage varied widely, and the unmet need ranged from 25\% to $96 \%$. Fifty percent of women have an unmet need for family planning services among all women who wish to delay the future pregnancy [13]. It is also higher among women in developing regions, particularly in low-income countries of subSaharan Africa [14]. Even though the postpartum modern contraceptive is critical, studies suggest that its use varies widely across geographical regions of low- and middleincome countries [13]. Therefore, this systematic review and meta-analysis was aimed at estimating the pooled prevalence of modern family planning among postpartum women in low-income countries of sub-Saharan Africa.

\section{Materials and Methods}

2.1. Study Design and Protocol Registration. A systematic review and meta-analysis of published and unpublished studies were used. This systematic review and meta-analysis were carried out in accordance with the recommendation of the Preferred Reporting Items for Systematic Reviews and MetaAnalyses (PRISMA) 2015 statement [15]. The protocol of this systematic review and meta-analysis has been registered on the International Prospective Register of Systematic Reviews (PROSPERO), registration number CRD42020160612 avail- able from https://www.crd.york.ac.uk/prospero/display_ record.php?.

\subsection{Criteria for Eligibility}

2.2.1. Inclusion Criteria. Articles were included in this systematic review and meta-analysis if they fulfilled all inclusion criteria:

(i) Publication condition: published studies, unpublished studies, and $\mathrm{PhD}$ dissertations which reported the outcome of interest were considered

(ii) Outcome of interest: studies reported data on the prevalence of postpartum modern contraceptives or/and their determinants were considered

(iii) Publication year: published articles between January1/2010 and January 1/2020 were included

(iv) Study design: observational studies (crosssectional)

(v) Study setting: all studies were conducted at the community or health institution level

(vi) Language: articles published in English and French languages were included

(vii) Study area: studies conducted in low-income countries of sub-Saharan Africa were included

(viii) Population: articles on postpartum women were considered. Studies with data on contraceptive use in the first 12 months postpartum period were included. Studies that included follow-up data after an extended postpartum period were included if the data could be disaggregated by month, which only includes data during the first 12 months postpartum

2.2.2. Exclusion Criteria. Articles which were not fully accessed after at least two email contacts of the primary author or failed to contact their primary authors were excluded.

2.3. Search Strategies. Relevant published studies were searched from the PubMed, HINARI, ScienceDirect, Wiley Library, ETH Library, and Cochrane Library electronic databases. Likewise, a search for grey literature was conducted using direct Google search and Google Scholar. Medical subject heading (MeSH), keywords, and thesaurus were used to identify selected PICO components. To combine search terms, Boolean operators ("OR," "AND," and "NOT") were used. The following keywords were used in the search: "postpartum family planning" OR "planification familiale postpartum" OR "postpartum contraceptive" OR "contraceptif post-partum” AND Prevalence OR Epidemiology OR utilization OR Utilisation OR use AND determinants OR déterminante OR "Factors associated" OR "facteurs associés" OR predictors OR prédicteurs. This review included studies published in English and French between January 1, 2010, and 
January 30,2020 . The search was carried out from 01 January 2020 to 01 March 2020.

2.4. Study Selection. All articles identified for the review were imported to EndNote X7, and duplicated studies were excluded. All studies were initially examined for inclusion based on information contained in the titles alone and abstracts. Then, a full-text review was performed by two independent reviewers (TT and TK). Cohen's kappa agreement test was done to test interrater reliability. The kappa coefficient was $k=0.667$ and $p<0.001$ with an asymptotic standard error of 0.124 , indicating the agreement between the two reviewers was substantial [16]. The PRISMA flow diagrams were used to summarize the selection procedure and process of the article [17].

2.5. Quality Appraisal. Two reviewers (TT and TK) independently assessed the risk of bias in the study. A modified version of the Newcastle-Ottawa Scale was used to appraise the quality of the studies [18]. The studies were divided into three categories: (0-4) low quality, (5-7) medium quality, and (810) high quality [19]. Any disagreement which arose between the two reviewers was solved through discussion and reached to consensus by involving a third reviewer. Those studies with medium (satisfying 50\%) and high qualities were included for analysis.

2.6. Data Extraction. Data was extracted from all articles that met the inclusion criteria using Microsoft Excel spreadsheet. The data extraction tool was adopted from the Joanna Briggs Institute (JBI) data extraction checklist for observational studies [20]. The data extraction tool was pretested in 10 randomly selected studies. The extracted data was entered into a Microsoft Excel spreadsheet before being exported to the STATA 14 software. All relevant information was extracted by two independent reviewers (TT and TK), while the authors' name, study area, and journal were blinded. The discrepancies were solved through discussion. In the case of incomplete data on constructing two-by-two tables, the reported odds ratio with its confidence interval (OR; 95\% $\mathrm{CI})$ was used. Incomplete data was requested for by contacting the authors.

2.7. Data Analysis, Publication Bias, and Heterogeneity. STATA Version 14 (software) was used for data entry and analysis. Funnel plots and Egger's and Begg's tests were used to examine the possible risk of publication bias. Statistical heterogeneity was checked by using the Cochran's $Q$ test and the $I^{2}$ test, which shows the percentage of total variation across the studies due to heterogeneity rather than chance. The $I^{2}$ statistics of below $25 \%$ are low heterogeneity, 25$50 \%$ is moderate heterogeneity, $51-75 \%$ is substantial heterogeneity, and above $75 \%$ is considerable heterogeneity [21]. A $p$ value $<0.05$ was used to declare heterogeneity. In the case of heterogeneity, the random effect (DerSimonian and Laird) model was used to estimate the pooled prevalence of postpartum modern contraceptive use. Forest plot and odds ratios with their 95\% CI were used to present the pooled effect sizes. Subgroup analysis was conducted by region and study setting. A forest plot was constructed for each variable. Metare- gression models were used to explore the relationship between the study-specific effect size and the study level covariates. Sensitivity analysis was used to measure the predictive power by excluding a single study.

\section{Results and Discussion}

3.1. Study Selection. A total of 743 articles were searched through the electronic databases: 301 articles from PubMed, 43 articles from HINARI, 122 articles from ScienceDirect, 51 from Cochrane Library, 219 from Google Scholar, 4 articles from Wiley Library, and again 4 articles from ETH Library. From these, 134 articles were excluded due to duplications, while the remaining 609 articles were reserved for further screening. Of these remaining articles, 320 and 241 articles were excluded by their titles and abstracts, respectively. A total of 48 full-text articles were assessed for eligibility criteria. Finally, 33 articles with appropriate quality were included in the final systematic review and meta-analysis. Furthermore, the PRISMA flow diagram was used to summarize the selection procedure (Additional file 1).

3.2. Characteristics of Original Studies. As described in Table 1, these 33 original articles were included in this systematic review and meta-analysis. The selected articles were published from 2010 to 2020. Two of them were unpublished articles [22, 23]. Regarding study design, all studies are crosssectional in nature. The sample size of the included studies ranged from 248 (in Ethiopia, Eastern Africa) [10] to 3617 (in Malawi, Eastern Africa) [24]. In this study, 27,128 postpartum women were involved. Among the 33 included studies, most of the studies (87.88\%) were from Eastern African countries. Out of 33 studies, 21 studies were conducted both in urban and rural settings [22-42], eight were in urban [10, 43-49], and four [50-53] were done in rural settings.

Among the 26 studies that reported the mean age of postpartum women, it ranged from 25 [31] to 30.8 years [33]. The highest and lowest prevalences of postpartum modern contraceptive use ( $80.3 \%$ and $3.41 \%$, respectively) were reported from Addis Ababa, Ethiopia [44], and Rural Guinea, West Africa [51], respectively. The response rate of included studies ranges from $94.3 \%$ [51] to $100 \%[10,23,30,38,45,50]$. Finally, the quality score of the included studies ranges from 6 up to 9 out of 10 points. Further descriptions and characteristics of the studies selected for this systematic review and meta-analysis are presented in Table 1 .

3.3. Quality of Included Studies in the Systematic Review. Modified versions of the Newcastle-Ottawa Scale [18] were used to assess the quality of the selected articles. Quality scores were defined based on the presence of sample representativeness, sample size, reporting of response rate, ascertainment of study outcomes, control of confounder, and quality of descriptive statistics reporting. Regarding the quality score of the included studies, 16 of the 33 studies had high quality (8-10 points) and the remaining 17 studies were medium-level quality (5-7 points).

3.4. Heterogeneity and Publication Bias. Statistical heterogeneity was checked by using Cochran's $Q$ test and the $I^{2}$ 
TABLE 1: Descriptive summary of 33 studies included in the systematic review and meta-analysis of postpartum modern contraceptive use among women of reproductive age (15-49 years) group in their first 12 months after delivery in low-income countries of sub-Saharan Africa, 2020

\begin{tabular}{|c|c|c|c|c|c|c|c|c|}
\hline Author & $\begin{array}{l}\text { Publication } \\
\text { year }\end{array}$ & Country & Study design & $\begin{array}{l}\text { Sample } \\
\text { size }\end{array}$ & $\begin{array}{l}\text { Women's } \\
\text { mean age }\end{array}$ & $\begin{array}{l}\text { Response } \\
\text { rate }(\%)\end{array}$ & $\begin{array}{l}\text { Prevalence } \\
(95 \% \mathrm{CI})\end{array}$ & $\begin{array}{c}\text { Quality score } \\
\text { (10 pts) }\end{array}$ \\
\hline Abera et al. [25] & 2015 & Ethiopia & $\begin{array}{c}\text { Cross- } \\
\text { sectional }\end{array}$ & 703 & 27.2 & 99.7 & $\begin{array}{c}48.36(48.22- \\
48.5)\end{array}$ & 8 \\
\hline Abraha et al. [50] & 2018 & Ethiopia & $\begin{array}{l}\text { Cross- } \\
\text { sectional }\end{array}$ & 1109 & 28.7 & 100 & $\begin{array}{c}38.32(38.24 \\
38.4)\end{array}$ & 7 \\
\hline Abraha et al. [26] & 2017 & Ethiopia & $\begin{array}{l}\text { Cross- } \\
\text { sectional }\end{array}$ & 590 & 27.4 & 98.2 & $\begin{array}{c}47.96(47.8- \\
48.13)\end{array}$ & 8 \\
\hline $\begin{array}{l}\text { Ashebir and Tadesse } \\
\text { [27] }\end{array}$ & 2020 & Ethiopia & $\begin{array}{l}\text { Cross- } \\
\text { sectional }\end{array}$ & 681 & 30.26 & 99.3 & $\begin{array}{c}20.7(20.59- \\
20.82)\end{array}$ & 8 \\
\hline Belda et al. [28] & 2019 & Ethiopia & $\begin{array}{l}\text { Cross- } \\
\text { sectional }\end{array}$ & 505 & 27.67 & 98.0 & $\begin{array}{c}14.26(14.12, \\
14.93)\end{array}$ & 7 \\
\hline Belete et al. [29] & 2019 & Ethiopia & $\begin{array}{l}\text { Cross- } \\
\text { sectional }\end{array}$ & 400 & 26.82 & 99.5 & $\begin{array}{c}58.5(58.26- \\
58.74)\end{array}$ & 8 \\
\hline Bwazi et al. [31] & 2014 & Malawi & $\begin{array}{l}\text { Cross- } \\
\text { sectional }\end{array}$ & 383 & 25 & 100 & $\begin{array}{c}74.67(74.45- \\
74.9)\end{array}$ & 6 \\
\hline Camara et al. [51] & 2018 & Guinea & $\begin{array}{l}\text { Cross- } \\
\text { sectional }\end{array}$ & 381 & 25.2 & 94.3 & $\begin{array}{c}3.41(3.32- \\
3.5)\end{array}$ & 6 \\
\hline Dasgupta et al. [52] & 2016 & Malawi & $\begin{array}{l}\text { Cross- } \\
\text { sectional }\end{array}$ & 442 & 26.0 & $\mathrm{NR}$ & $\begin{array}{c}28.5(28.3- \\
28.7)\end{array}$ & 6 \\
\hline Demie et al. [10] & 2018 & Ethiopia & $\begin{array}{l}\text { Cross- } \\
\text { sectional }\end{array}$ & 248 & 27.40 & 100 & $\begin{array}{c}33.06(32.69- \\
33.44)\end{array}$ & 7 \\
\hline Dulli et al. [32] & 2010 & Madagascar & $\begin{array}{l}\text { Cross- } \\
\text { sectional }\end{array}$ & 840 & 26.8 & 100 & $\begin{array}{c}17.26(17.17- \\
17.35)\end{array}$ & 6 \\
\hline $\begin{array}{l}\text { Gebremariam and } \\
\text { Gebremariam [33] }\end{array}$ & 2017 & Ethiopia & $\begin{array}{l}\text { Cross- } \\
\text { sectional }\end{array}$ & 599 & 30.8 & 99 & $\begin{array}{c}68.11(67.96- \\
68.27)\end{array}$ & 8 \\
\hline Gebremedhin et al. [44] & 2018 & Ethiopia & $\begin{array}{l}\text { Cross- } \\
\text { sectional }\end{array}$ & 803 & NR & 94.6 & $\begin{array}{c}80.32(80.22- \\
80.42)\end{array}$ & 8 \\
\hline Gejo et al. [45] & 2019 & Ethiopia & $\begin{array}{l}\text { Cross- } \\
\text { sectional }\end{array}$ & 368 & 29.12 & 100 & $\begin{array}{c}73.91(73.68- \\
74.15)\end{array}$ & 9 \\
\hline Getachew [22] & 2016 & Ethiopia & $\begin{array}{l}\text { Cross- } \\
\text { sectional }\end{array}$ & 420 & 27.5 & 99.7 & $\begin{array}{c}47.38(47.15- \\
47.61)\end{array}$ & 7 \\
\hline Gizaw et al. [34] & 2017 & Ethiopia & $\begin{array}{l}\text { Cross- } \\
\text { sectional }\end{array}$ & 829 & 27.53 & 98.2 & $\begin{array}{c}46.68(46.56- \\
46.80)\end{array}$ & 8 \\
\hline Asires et al. [43] & 2017 & Ethiopia & $\begin{array}{l}\text { Cross- } \\
\text { sectional }\end{array}$ & 833 & 27.3 & 98.6 & $\begin{array}{c}65.67(65.55- \\
65.78)\end{array}$ & 7 \\
\hline Hounkponou et al. [46] & 2019 & Benin & $\begin{array}{l}\text { Cross- } \\
\text { sectional }\end{array}$ & 453 & 27.1 & 98.5 & $\begin{array}{c}13.02(12.9- \\
13.17)\end{array}$ & 8 \\
\hline Jaleta et al. [35] & 2019 & Ethiopia & $\begin{array}{l}\text { Cross- } \\
\text { sectional }\end{array}$ & 820 & 28 & 95.6 & $\begin{array}{c}37.19(37.08- \\
37.31)\end{array}$ & 8 \\
\hline Kaydor et al. [36] & 2018 & Liberia & $\begin{array}{l}\text { Cross- } \\
\text { sectional }\end{array}$ & 378 & NR & 100 & $\begin{array}{c}11.90(11.74- \\
12.07)\end{array}$ & 7 \\
\hline Keogh et al. [37] & 2015 & Tanzania & $\begin{array}{l}\text { Cross- } \\
\text { sectional }\end{array}$ & 2162 & NR & NR & $\begin{array}{c}34.32(34.28- \\
34.36)\end{array}$ & 6 \\
\hline Berta et al. [30] & 2018 & Ethiopia & $\begin{array}{l}\text { Cross- } \\
\text { sectional }\end{array}$ & 404 & $\mathrm{NR}$ & 100 & $\begin{array}{c}45.79(45.55- \\
46.03)\end{array}$ & 8 \\
\hline Mengesha et al. [38] & 2015 & Ethiopia & $\begin{array}{l}\text { Cross- } \\
\text { sectional }\end{array}$ & 899 & 28.3 & 100 & $\begin{array}{c}10.34(10.28- \\
10.41)\end{array}$ & 8 \\
\hline Nigussie et al. [39] & 2016 & Ethiopia & $\begin{array}{l}\text { Cross- } \\
\text { sectional }\end{array}$ & 545 & 31 & 98 & $\begin{array}{c}12.29(12.17- \\
12.41)\end{array}$ & 8 \\
\hline Palamuleni [24] & 2012 & Malawi & $\begin{array}{l}\text { Cross- } \\
\text { sectional } \\
\text { (DHS) }\end{array}$ & 3617 & NR & NR & $\begin{array}{c}49.49(49.46- \\
49.52)\end{array}$ & 7 \\
\hline
\end{tabular}


TABLe 1: Continued.

\begin{tabular}{|c|c|c|c|c|c|c|c|c|}
\hline Author & $\begin{array}{c}\text { Publication } \\
\text { year }\end{array}$ & Country & Study design & $\begin{array}{l}\text { Sample } \\
\text { size }\end{array}$ & $\begin{array}{l}\text { Women's } \\
\text { mean age }\end{array}$ & $\begin{array}{c}\text { Response } \\
\text { rate }(\%)\end{array}$ & $\begin{array}{c}\text { Prevalence } \\
(95 \% \mathrm{CI})\end{array}$ & $\begin{array}{c}\text { Quality score } \\
\text { (10 pts) }\end{array}$ \\
\hline Rutaremwa et al. [40] & 2015 & Uganda & $\begin{array}{c}\text { Cross- } \\
\text { sectional } \\
\text { (DHS) }\end{array}$ & 3298 & 29.7 & 100 & $\begin{array}{c}27.71(27.69- \\
27.74)\end{array}$ & 7 \\
\hline Samuel [47] & 2016 & $\begin{array}{l}\text { South } \\
\text { Sudan }\end{array}$ & $\begin{array}{c}\text { Cross- } \\
\text { sectional }\end{array}$ & 295 & 25.4 & 100 & $\begin{array}{c}6.10(5.94- \\
6.26)\end{array}$ & 7 \\
\hline Sileo et al. [53] & 2015 & Uganda & $\begin{array}{c}\text { Cross- } \\
\text { sectional }\end{array}$ & 258 & 25.85 & 100 & $\begin{array}{c}25.19(24.86- \\
25.52)\end{array}$ & 7 \\
\hline Taye et al. [48] & 2019 & Ethiopia & $\begin{array}{c}\text { Cross- } \\
\text { sectional }\end{array}$ & 546 & 27.57 & 97 & $\begin{array}{c}63.0(62.83- \\
63.18)\end{array}$ & 8 \\
\hline Tedla [49] & 2017 & Ethiopia & $\begin{array}{c}\text { Cross- } \\
\text { sectional }\end{array}$ & 623 & 27.5 & 98.7 & $\begin{array}{c}50.08(49.92- \\
50.24)\end{array}$ & 8 \\
\hline Tegegn et al. [41] & 2017 & Ethiopia & $\begin{array}{l}\text { Cross- } \\
\text { sectional }\end{array}$ & 382 & 28 & 99.7 & $\begin{array}{c}54.71(54.46- \\
54.97)\end{array}$ & 8 \\
\hline Zivich et al. [42] & 2018 & DRC & $\begin{array}{c}\text { Cross- } \\
\text { sectional }\end{array}$ & 522 & NR & 95.8 & $6.9(6.80-70)$ & 7 \\
\hline Zzimbe [23] & 2015 & Uganda & $\begin{array}{c}\text { Cross- } \\
\text { sectional }\end{array}$ & 1792 & NR & 100 & $\begin{array}{c}19.47(19.43- \\
19.52)\end{array}$ & 7 \\
\hline
\end{tabular}

Note: DHS: demographic health survey; NR: not reported.

test. In this analysis, considerable heterogeneity was observed across the included studies and detected by the Cochran $Q$ test $(Q$ test $p<0.001)$ and $I^{2}$ statistics $\left(I^{2}=100 \%\right) \quad$ (Figure 1). Therefore, DerSimonian and Laird's random effect model was used. Publication bias was checked by funnel plots. The shape of the funnel plots indicates a slightly asymmetrical distribution (Figure 2). Moreover, to ascertain the funnel plot, Begg's and Egger's objective tests were conducted. Begg's and Egger's test results showed that there was no statistically significant publication bias across the included studies $(p=0.69$ and $p=0.50$, respectively).

3.5. Meta-Analysis. In this meta-analysis, considerable heterogeneity $\left(I^{2}=100 \%, p<0.001\right)$ was observed across the studies. Therefore, a random effect meta-analysis model was used to estimate the pooled effect of postpartum contraceptive use. As shown in the forest plot, the results of 33 included studies indicated that the pooled prevalence of postpartum contraceptive use in low-income countries of subSaharan Africa was 37.41\%, 95\% CI: (31.35, 43.48\%) (Figure 1).

3.6. Subgroup Analysis. Subgroup analysis was performed based on the regions where the studies were conducted, sample size, study setting, and year of publication of the studies to assess possible causes of considerable heterogeneity. Based on subgroup analysis, Eastern Africa had the highest prevalence of postpartum contraceptive use $(41.36 \%, 95 \% \mathrm{CI}$ : (35.2, 47.52)), followed by Western Africa (9.45\%, 95\% CI: $(2.69,16.2))$ and others (central Africa 6.9\%). Regarding the study setting, the prevalence of postpartum contraceptive utilization was $40.97 \%$ (95\% CI: $(33.38,48.55))$ among studies conducted at the community level (Table 2). A prevalence of $(38.66 \%, 95 \%$ CI: $(28.4,48.9))$ was observed in studies which have been published since 2016 (Table 2). However, the results of the subgroup analysis indicated that the source of considerable heterogeneity was not because of the study regions, sample size, study setting, and year of publication of the studies.

3.7. Determinants of Postpartum Modern Contraceptive Use in Low-Income Countries of Sub-Saharan Africa. Of the 33 studies identified, 31 studies were included to identify determinants of postpartum modern contraceptives. The two studies [24, 31] were excluded because of insufficient data to extract two-by-two tables, and the reported odds ratio does not have a confidence interval. Metaregression was performed for all selected determinants to determine the possible sources of variation, but there was no statistical significance. As a result, to determine the associations, a random effect model was computed (Figures 3(a)-3(f)).

3.7.1. Association between a Mother's Educational Status and Postpartum Modern Contraceptive Use. To determine the association of a mother's educational status with postpartum modern contraceptive use, 18 studies were included. The findings of the studies indicated that those mothers who had secondary and above level of education were 2.09 times more likely to use postpartum contraceptives (AOR 2.09, 95\% CI: $(1.52,2.86))$, compared to those who had primary education and below.

In this analysis, the test statistics indicated that considerable heterogeneity $\left(I^{2}=89.7 \%\right.$ and $\left.p=0.000\right)$ was presented across the included studies. To explore this heterogeneity, a sensitivity analysis was done and there was no significant change in the overall results of OR. In addition, Begg's and Egger's tests revealed the absence of statistically significant publication bias ( $p=0.058$ and $p=0.664$, respectively). 


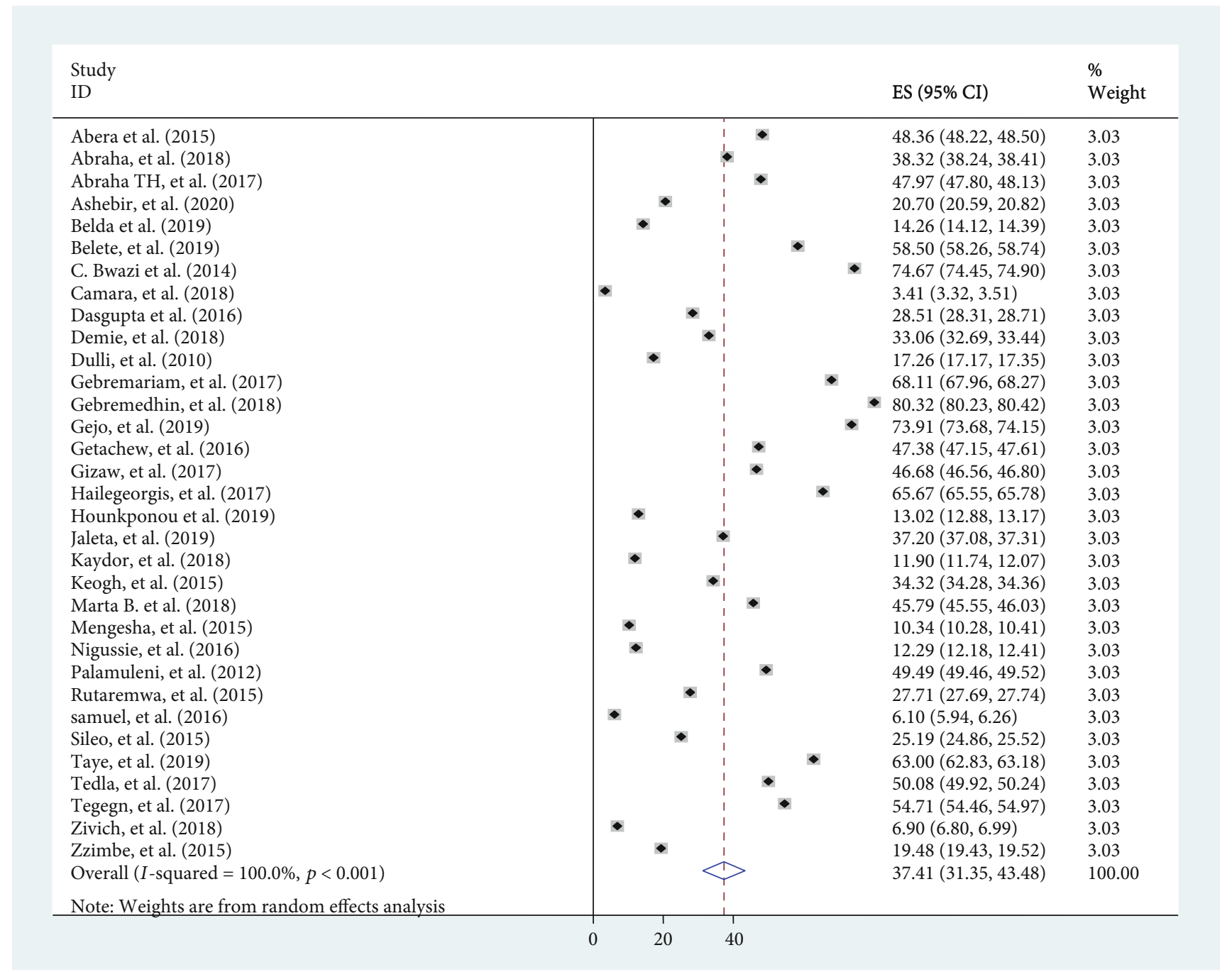

FIGURE 1: Forest plot of the pooled prevalence of postpartum modern contraceptive use with its $95 \%$ confidence interval among women of the reproductive age (15-49 years) group in their first 12 months after delivery in low-income countries of sub-Saharan Africa, 2020.

3.7.2. Resumption of Menses and Use of Postpartum Modern Contraceptives. A total of 15 studies [22, 25-30, 34, 42-46, $48,49]$ were included. The findings revealed that women who experienced menstruation again after giving birth were four times more likely to use postpartum contraception than mothers who experienced amenorrhea in the postpartum period (AOR: 3.98, 95\% CI: $(2.62,6.03)$ ). Considerable heterogeneity was detected $\left(I^{2}=93.3 \% ; \quad p=0.000\right)$ (Figure 3(b)). The results of sensitivity analysis indicate that there is no significant change in the overall results of OR. The results of Begg's and Egger's tests showed that there was no statistically significant publication bias across 15 studies $(p=0.69$ and $p=0.50$, respectively).

3.7.3. Partner Discussion and Use of Postpartum Modern Contraceptives. The analysis included 14 studies [22, 26-28, $30,34,35,39,41,43,46,47,49,53]$. Studies revealed that discussing with the partner was significantly associated with the use of PPFP (AOR 3.68, 95\% CI: $(1.96,6.89)$ ) (Figure 3(c)). The selected studies exhibited considerable heterogeneity $\left(I^{2}=95.3 \%\right.$ and $\left.p<0.001\right)$. As a result, a random effect meta-analysis was employed. Publication bias was checked by using Begg's test $(p=0.125)$ and Egger's test $(p=0.213)$ which revealed that there was no evidence of publication bias.

3.7.4. Having Knowledge of Modern Contraceptive Methods. From the meta-analysis of eleven $[22,26-30,35,36,39,43$, 49] studies, knowledge of modern contraceptive methods was significantly associated with the use of PPFP (AOR: 5.65, 95\% CI: 3.58, 8.93)). The overall heterogeneity was $I^{2}$ $=77.3 \%$ and $p<0.001$. As a result, a random effect metaanalysis was used. Publication bias assessed by using Begg's and Egger's tests revealed that there was a low possibility of publication bias with $p$ values of 0.28 and 0.08 , respectively.

3.7.5. Having ANC Follow-Up. From the results of nine included studies [22, 25, 35, 39, 41, 43, 45, 48, 49], the heterogeneity test showed the presence of substantial heterogeneity $\left(I^{2}=52.8 \%\right)$. The pooled effect sizes of PPFP utilization among women who have attended at least one ANC visit were five times more likely (AOR; 5.10, 95\% CI: (3.57, 7.29)) to use PPFP compared to those women not having 


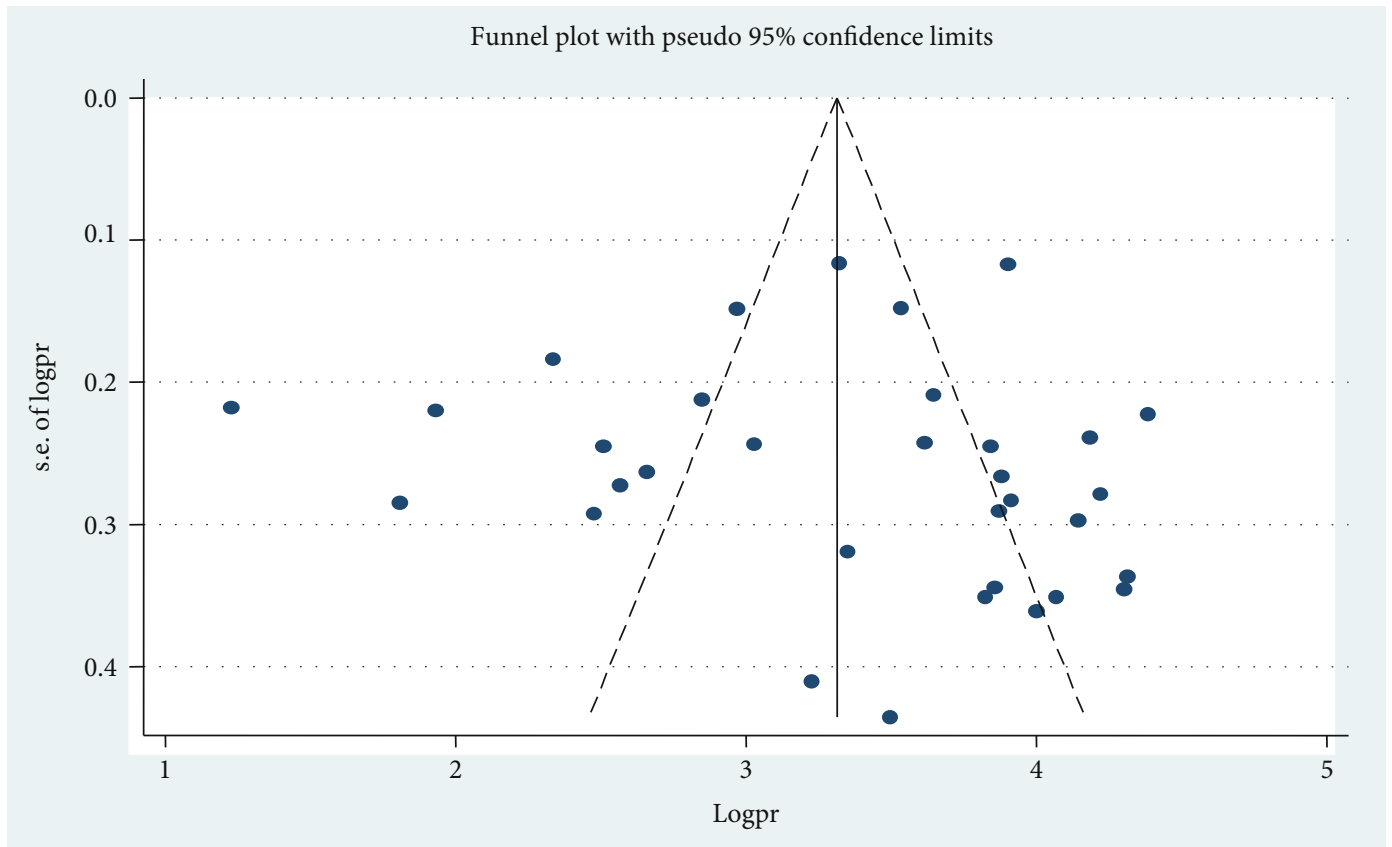

Figure 2: Funnel plot to check publication bias of the 33 included studies in low-income countries of sub-Saharan Africa, 2020.

TABLE 2: Subgroup analysis for prevalence of postpartum modern contraceptive use among women of reproductive age (15-49 years) group in their first 12 months after delivery in low-income countries of sub-Saharan Africa, $2020(n=33)$.

\begin{tabular}{|c|c|c|c|c|c|c|}
\hline Variable & Subgroup & Number of studies & Sample size & $I^{2}$ & ES $(95 \%$ CI $)$ & $p$ value \\
\hline \multirow{2}{*}{ Sample size* } & $\geq 822$ & 9 & 15,379 & $99.57 \%$ & $34.36(24.76,43.96)$ & $<0.001$ \\
\hline & $<822$ & 24 & 11,749 & $99.54 \%$ & $38.56(27.71,49.41)$ & $<0.001$ \\
\hline \multirow{2}{*}{ Publication year } & $2010-2015$ & 9 & 13,952 & $99.52 \%$ & $34.09(24.46,43.72)$ & $<0.001$ \\
\hline & 2016-2020 & 24 & 13,176 & $99.58 \%$ & $38.66,(28.41,48.91)$ & $<0.001$ \\
\hline \multirow{2}{*}{ Study setting } & Community-based & 21 & 19,801 & $99.51 \%$ & $40.97(33.38,48.55)$ & $<0.001$ \\
\hline & Facility-based & 12 & 7327 & $99.56 \%$ & $31.2(19.4,42.99)$ & $<0.001$ \\
\hline
\end{tabular}

Note: *sample size for subgroup analysis categorized by taking the mean sample size.

ANC visits (Figure 3(e)). Begg's and Egger's tests for publication bias showed no statistical evidence of publication bias ( $p=0.75$ and $p=0.899$, respectively).

\subsubsection{Family Planning Counselling and the Use of Postpartum} Modern Contraceptives. Women who received family planning counselling during antenatal care were nearly six times more likely to use modern contraceptives in the postpartum period than those who did not $(\mathrm{AOR}=5.92,95 \% \mathrm{CI}$ : $(2.54$, 13.79)). The heterogeneity test showed that considerable heterogeneity was found $\left(I^{2}=93.2 \%, p=0.000\right)$ between the studies (Figure 3(f)). To reduce the random variation, a sensitivity analysis was done, but did not bring a significant change in the overall results of OR.

3.8. Discussion. In this systematic review and meta-analysis, the overall pooled prevalence of postpartum modern contraceptive use in low-income countries of SSA was 37.41\%. However, use varied regionally, with the highest prevalence of postpartum contraceptive use observed in Eastern Africa (41.36\%), followed by Western Africa (9.45\%) and Central Africa (6.9\%). This finding is in line with a meta-analysis conducted in low- and middle-income countries, which was found to be $41.2 \%$ [54]. However, the current finding is lower than studies done in lower middle-income countries of South Asia (Bangladesh, 53\%) [55], but higher than studies done in India $(23 \%, 25.4 \%)[56,57]$ and Pakistan $(24.6 \%)$ [58]. The possible explanations for this variation might be due to heterogeneous socioeconomic, sociodemographic, and cultural differences between the populations.

In our meta-analysis, higher educated mothers were more likely to use PPFP than mothers with a low education level. It is in line with studies conducted in low- and middle-income countries $[7,54]$. The possible explanations might be that when women's educational status increases, they will have better health care seeking behavior, they understand the benefits and disadvantages of contraceptives, and they will have the right information about fertility and contraception. Therefore, empowering maternal education helps them to make an informed decision on their fertility and for better maternal and child health.

Women who resumed menstruation after giving birth were more likely to use PPFP than mothers who experienced postpartum amenorrhea. This finding is comparable with the 
Educational status and the use of PPFP

Study

ID

Abera et al. (2015)

Abraha TH, et al. (2017)

Ashebir, et al. (2020)

Belete, et al. (2019)

Dasgupta et al. (2016)

Demie, et al. (2018)

Gejo, et al. (2019)

Gizaw, et al. (2017)

Hounkponou, et al. (2019)

Jaleta, et al. (2019)

Mengesha, et al. (2015)

Nigussie, et al. (2016)

Rutaremwa, et al. (2015)

samuel, et al. (2016)

Sileo, et al. (2015)

Taye, et al. (2019)

Tegegn, et al. (2017)

Zivich, et al. (2018)

Overall $(I$-squared $=89.7 \%, p<0.001)$

Note: Weights are from random effects analysis

$\begin{array}{ll}\% \\ \text { ES }(95 \% \mathrm{CI}) & \text { Weight }\end{array}$

$1.40(1.04,1.88)$

6.38

$1.25(0.91,1.73) \quad 6.31$

$2.25(1.23,4.13) \quad 5.41$

$1.94(1.28,2.93) \quad 6.06$

$1.37(0.88,2.12) \quad 5.97$

$2.43(1.39,4.24) \quad 5.59$

$0.34(0.21,0.54) \quad 5.89$

$4.10(1.05,16.07) \quad 2.99$

$2.83(1.62,4.95) \quad 5.58$

$1.51(1.07,2.13) \quad 6.27$

$11.20(7.01,17.89) \quad 5.88$

$12.12(5.14,28.59) \quad 4.51$

$2.44(2.06,2.90) \quad 6.63$

$6.20(1.99,19.33) \quad 3.60$

$2.31(1.31,4.08) \quad 5.55$

$1.23(0.86,1.75) \quad 6.22$

$1.73(1.15,2.60) \quad 6.08$

$1.31(0.65,2.63) \quad 5.09$

$2.09(1.52,2.86) \quad 100.00$

(a)

Figure 3: Continued. 
Forest plot of resumption of menses

Study

ID

Hounkponou, et al. (2019)

Zivich, et al. (2018)

Tedla, et al. (2017)

Gebremedhin, et al. (2018)

Getachew, et al. (2016)

Taye, et al. (2019)

Belda et al. (2019)

Marta B. et al. (2018)

Abera et al. (2015)

Gejo, et al. (2019)

Abraha TH, et al. (2017)

Belete, et al. (2019)

Ashebir, et al. (2020)

Asires, et al. (2017)

Gizaw, et al. (2017)

Overall $(I$-squared $=93.3 \%, p<0.001)$

Note: Weights are from random effects analysis $\begin{array}{ll}\% \\ \text { ES }(95 \% \text { CI }) & \text { Weight }\end{array}$

$3.00(1.71,5.27) \quad 6.39$

$2.01(0.97,4.17) \quad 5.92$

$2.67(1.93,3.69) \quad 6.93$

$0.47(0.33,0.66) \quad 6.88$

$3.51(2.28,5.40) \quad 6.72$

$3.67(2.55,5.30) \quad 6.86$

$7.77(4.48,13.46) \quad 6.43$

$5.57(3.61,8.60) \quad 6.71$

$9.74(6.87,13.79) \quad 6.89$

$9.90(5.68,17.27) \quad 6.41$

$6.47(4.40,9.50) \quad 6.82$

$3.58(2.36,5.43) \quad 6.75$

$2.98(2.03,4.36) \quad 6.82$

$4.33(3.19,5.88) \quad 6.97$

$8.49(5.04,14.30) \quad 6.50$

$3.98(2.62,6.03) \quad 100.00$

(b)

Figure 3: Continued. 


\begin{tabular}{l} 
Study \\
ID \\
\hline Hounkponou, et al. (2019)
\end{tabular}

(c)

Knowledge of modern contraceptive methods and PPFP

Study

ID

Tedla, et al. (2017)

Getachew, et al. (2016)

Belda et al. (2019)

Marta B. et al. (2018)

Nigussie, et al. (2016)

Abraha TH, et al. (2017)

Belete, et al. (2019)

Ashebir , et al. (2020)

Jaleta, et al. (2019)

Kaydor, et al. (2018)

Asires, et al. (2017)

Overall $(I$-squared $=77.3 \%, p<0.001)$

Note: Weights are from random effects analysis
ES $(95 \%$ CI)

$62.14(15.14,255.02)$
$8.42(5.13,13.83)$
$2.99(1.70,5.26)$
$4.38(2.25,8.50)$
$4.26(1.96,9.27)$
$7.56(2.24,25.46)$
$1.92(1.27,2.89)$
$12.62(3.07,51.98)$
$6.90(4.08,11.66)$
$3.68(1.22,11.09)$
$6.20(2.75,13.97)$
$5.65(3.58,8.93)$

(d)

Figure 3: Continued. 


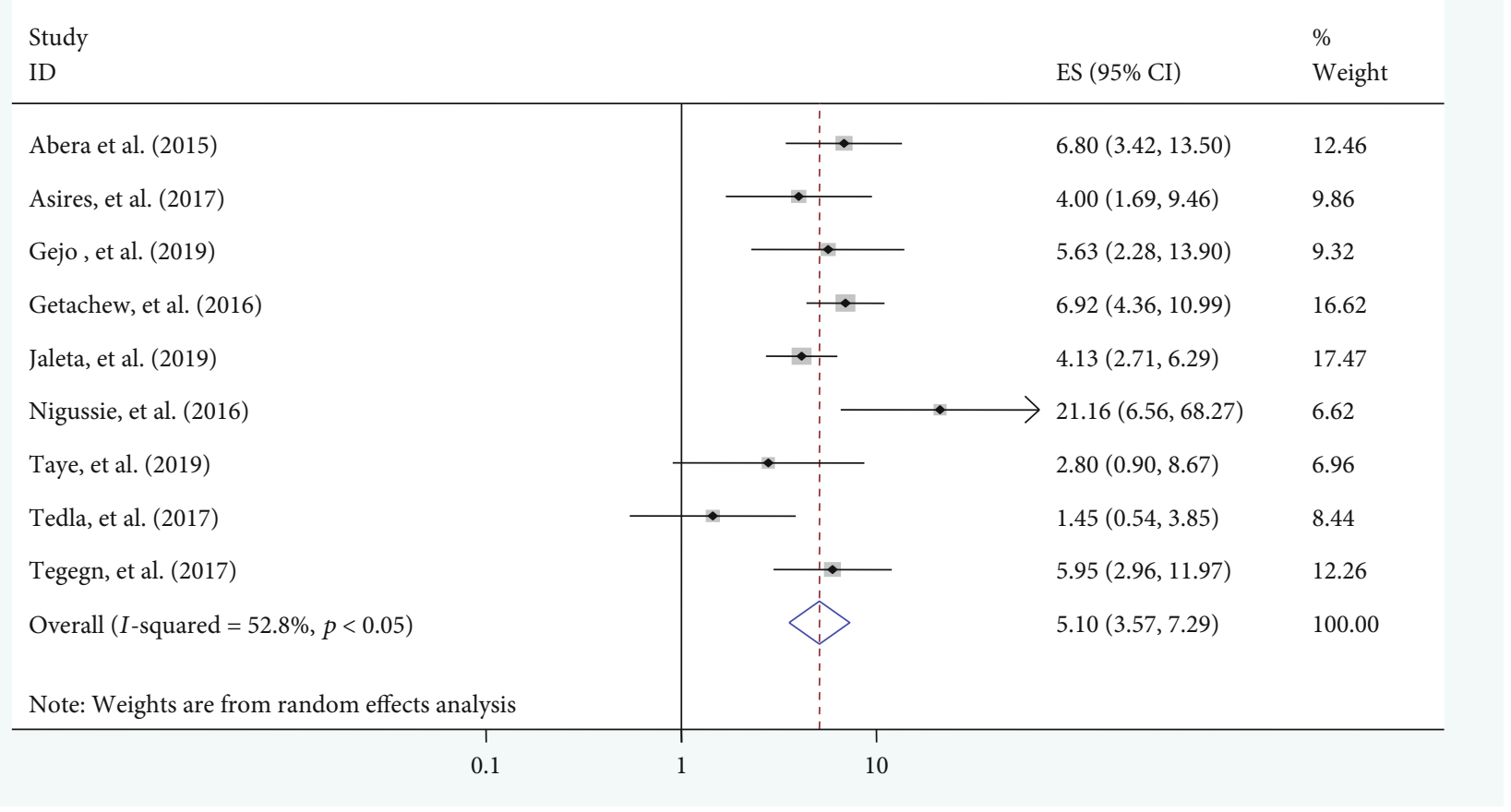

(e)

Forest plot of family planning counseling during ANC

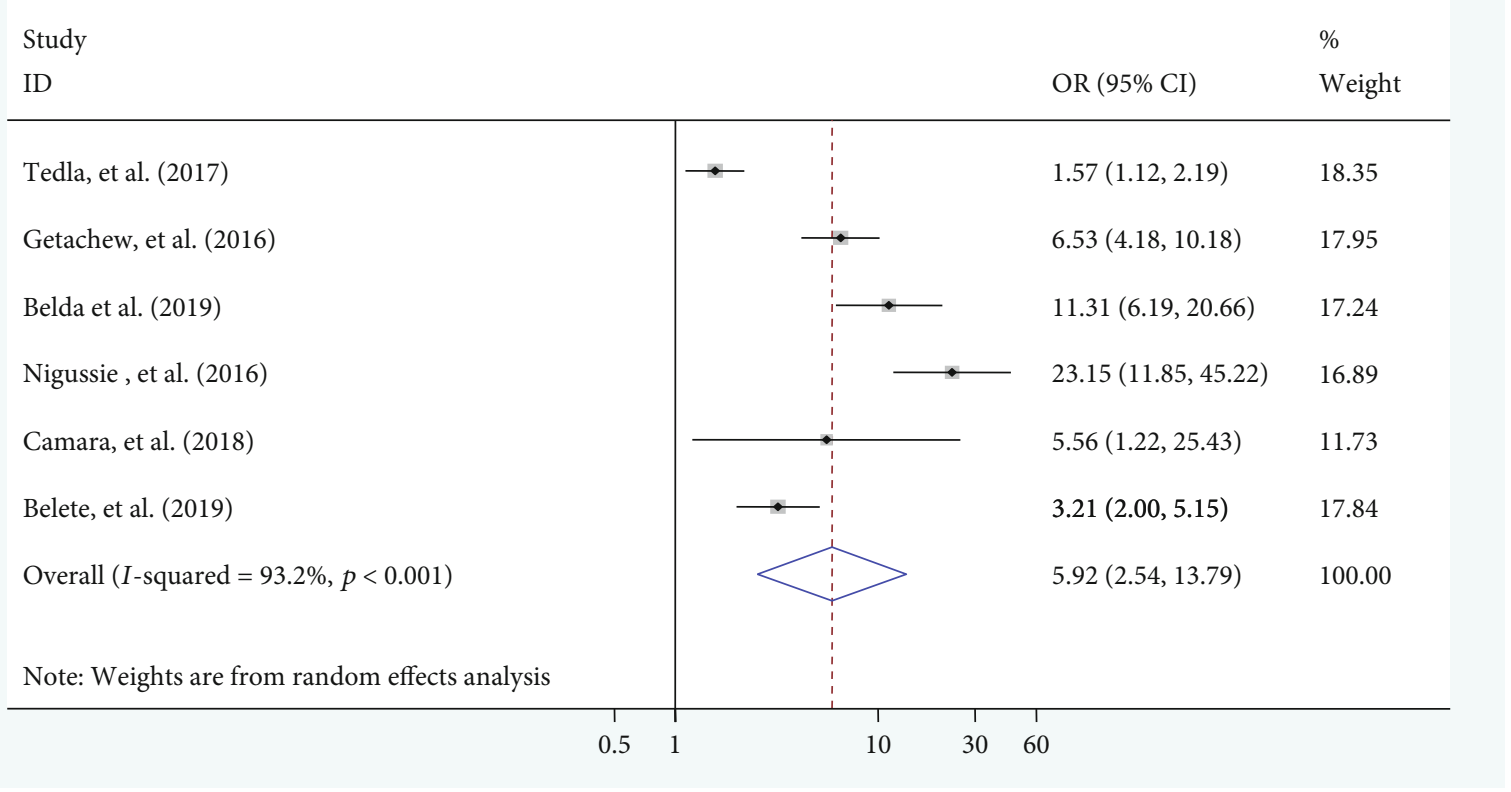

(f)

FIGURE 3: (a-f) Forest plot displaying the pooled odds ratio of the associations between the use of PPFP and its determinants: (a) mothers' educational status, (b) resumption of menses, (c) having partner discussion, (d) having knowledge of modern contraceptive methods, (e) having ANC follow-up, and (f) family planning counselling during ANC, in low-income countries of sub-Saharan Africa, 2020.

results of a meta-analysis conducted on PPFP [54, 55]. This is explained by the return of menstruation after delivery, which leads women to believe that once menstruation returns, the likelihood of becoming pregnant increases. Thus, they are more likely to start using a contraceptive.
However, many women do not begin using a contraceptive until menstruation has resumed. Therefore, it is required to educate women; biologically, a woman may ovulate before the first menstruation has returned following childbirth. 
Discussing with partners about PPFP was significantly associated with its use. Women who had a discussion with partners were more likely to use PPFP than their counterparts. The current finding is supported by a meta-analysis conducted in low- and middle-income countries [54]. This suggests that male involvement and support with family planning helps women to adopt more convenient methods with confidence.

In this finding, women who had knowledge of PPFP were more likely to use PPFP than those who did not. It is in line with the findings of a meta-analysis [54], but studies conducted in Malawi [31] showed that having knowledge of PPFP services is not significantly associated with the use of PPFP. Therefore, the pooled effect of this finding suggests having knowledge about modern contraceptives is a significant input to adopting family planning services. Therefore, creating awareness and promoting knowledge relating to modern contraceptives is required.

Women who had at least one ANC visit during pregnancy were more likely to use postpartum contraception than women who did not have an ANC visit. In contrast to the findings of the other included studies, ANC utilization was not statistically significant with the use of PPFP in a study conducted in North Gondar, Ethiopia [38]. This finding is in line with USAID findings from 17 countries and USAID DHS Comparative Reports $[6,55]$. The possible explanation for this finding might be that women who used ANC had more exposure to information on birth spacing and complications of the short birth interval for both the mother and newborn.

Women who received proper family planning counselling during antenatal care were more likely to utilize postpartum contraceptives. However, the findings of a study conducted in South Africa [59] contradict this; it shows that contraceptive counselling during antenatal visits could have no impact on contraceptive use, whereas the current finding is in line with a meta-analysis conducted in low- and middleincome countries [54]. This might be due to frequent and proper counselling provided by health care providers about contraceptive use and the risk of closely spaced pregnancies. Focused ANC incorporates ANC counselling sessions. Due to this, the ANC providers also give more attention to family planning counselling; this may be another possible justification for this finding.

3.9. Strengths and Limitations. We used extensive and comprehensive search strategies systematically from multiple databases. Published and unpublished studies and grey literature were included and evaluated for methodological quality using a standardized tool. However, this review represented three regions of SSA, but the majority of studies were obtained from the East Africa region. Therefore, the results may not be strongly representative for the other regions due to the small number of studies included.

\section{Conclusions}

Postpartum contraceptive utilization is low and not optimal compared to the global recommendation on postpartum family planning. A global increase in postpartum contraceptive use can help in reducing maternal and child mortality and improving the lives of women and their families. Secondary and higher education levels, resumption of menstruation, discussion with husbands about the use of PPFP, knowledge of modern contraceptive methods, and use of maternal health services (ANC follow-up and family planning counseling during ANC) were all significantly associated with the use of modern postpartum contraceptives.

\section{Data Availability}

All relevant datasets used and/or analyzed are available upon reasonable request to the corresponding author.

\section{Conflicts of Interest}

The authors declared that we have no competing interests regarding the publication of this paper.

\section{Authors' Contributions}

All authors have contributed to this study. TT contributed to the conception and design of the study, protocol preparation and registration, and search articles and extraction of data; conducted all analyses and interpretation of data; drafted the manuscript; and acted as the corresponding author. GG contributed to the design of the study, reviewing and revising the paper, providing detailed comments, editing the article, and drafting of the manuscript. GE contributed to the design of the study, reviewing and revising the paper, providing detailed comments, editing the article, and drafting of the manuscript. TK participated in data extraction and search for the article and assisted in drafting the manuscript. All authors read and approved the final manuscript.

\section{Acknowledgments}

The authors would like to thank the School of Public Health, College of Medicine and Health Sciences, Wollo University (Ethiopia) for nonfinancial support and for all lecturers, assisting professors, and family members (especially Kelem Gebre) who helped us with their support, valuable comments, and guidance throughout this work.

\section{Supplementary Materials}

Additional file 1: Preferred Reporting Items for Systematic Reviews and Meta-Analyses: the PRISMA statement 2009 checklist. (Supplementary Materials)

\section{References}

[1] WHO, Programming strategies for postpartum family planning, WHO, Geneva, Switzerland, 2013, https://www.who .int/reproductivehealth/publications/family_planning/ppfp_ strategies/en/.

[2] A. Welsh, Best practice in postpartum family planning, Royal College of Obstetricians and Gynaecologists, Regent's Park, London, 2015. 
[3] M. E. Gaffield, S. Egan, and M. Temmerman, "It's about time: WHO and partners release programming strategies for postpartum family planning," Global Health: Science and Practice, vol. 2, no. 1, pp. 4-9, 2014.

[4] WHO, Report of a WHO technical consultation on birth spacing, WHO, Geneva, 2005, https://www.who.int/maternal_ child_adolescent/documents/birth_spacing05/en/.

[5] J. DaVanzo, L. Hale, A. Razzaque, and M. Rahman, "Effects of interpregnancy interval and outcome of the preceding pregnancy on pregnancy outcomes in Matlab, Bangladesh," BJOG, vol. 114, no. 9, pp. 1079-1087, 2007.

[6] W. Winfrey and K. Rakesh, Use of family planning in the postpartum period, ICF International, 2014 , http://dhsprogram.com/pubs/pdf/CR36/CR36.pdf.

[7] S. Hounton, W. Winfrey, A. J. D. Barros, and I. Askew, "Patterns and trends of postpartum family planning in Ethiopia, Malawi, and Nigeria: evidence of missed opportunities for integration," Global Health Action, vol. 8, no. 1, p. 29738, 2015.

[8] J. Cleland, A. Conde-Agudelo, H. Peterson, J. Ross, and A. Tsui, "Contraception and health," The Lancet, vol. 380, no. 9837, pp. 149-156, 2012.

[9] S. Eliason, F. Baiden, G. Quansah-Asare et al., "Factors influencing the intention of women in rural Ghana to adopt postpartum family planning," Reproductive Health, vol. 10, no. 1, p. 34, 2013.

[10] T. G. Demie, T. Demissew, T. K. Huluka, D. Workineh, and H. G. Libanos, "Postpartum family planning utilization among postpartum women in public health institutions of Debre Berhan town, Ethiopia," Journal of Women's Health Care, vol. 7, no. 2, 2018.

[11] N. J. Kassebaum, A. Bertozzi-Villa, M. S. Coggeshall et al., "Global, regional, and national levels and causes of maternal mortality during 1990-2013: a systematic analysis for the Global Burden of Disease Study 2013," The Lancet, vol. 384, no. 9947, pp. 980-1004, 2014.

[12] Z. Moore, A. Pfitzer, R. Gubin, E. Charurat, L. Elliott, and T. Croft, "Missed opportunities for family planning: an analysis of pregnancy risk and contraceptive method use among postpartum women in 21 low- and middle-income countries," Contraception, vol. 92, no. 1, pp. 31-39, 2015.

[13] O. Pasha, S. S. Goudar, A. Patel et al., "Postpartum contraceptive use and unmet need for family planning in five lowincome countries," Reproductive Health, vol. 12, no. S2, p. S11, 2015.

[14] S. Singh and J. E. Darroch, Adding it up: costs and benefits of contraceptive services, Guttmacher Institute and UNFPA, 2012, http://www.guttmacher.org/pubs/AIU-2012-estimates.pdf.

[15] PRISMA-P Group, D. Moher, L. Shamseer et al., "Preferred reporting items for systematic review and meta-analysis protocols (PRISMA-P) 2015 statement," Systematic Reviews, vol. 4, no. 1, p. 1, 2015.

[16] A. J. Viera and J. M. Garrett, "Understanding interobserver agreement: the kappa statistic," Family Medicine, vol. 37, no. 5, pp. 360-363, 2005.

[17] D. Moher, A. Liberati, J. Tetzlaff, D. G. Altman, and PRISMA Group, "Preferred reporting items for systematic reviews and meta-analyses: the PRISMA statement," Annals of internal medicine, vol. 151, no. 4, pp. 264-269, 2009.

[18] C. K.-L. Lo, D. Mertz, and M. Loeb, "Newcastle-Ottawa Scale: comparing reviewers' to authors' assessments," BMC Medical Research Methodology, vol. 14, no. 1, p. 45, 2014.
[19] A. Stang, "Critical evaluation of the Newcastle-Ottawa Scale for the assessment of the quality of nonrandomized studies in meta-analyses," European Journal of Epidemiology, vol. 25, no. 9, pp. 603-605, 2010.

[20] M. D. Peters, C. M. Godfrey, P. McInerney, C. B. Soares, H. Khalil, and D. Parker, "The Joanna Briggs Institute reviewers' manual 2015: methodology for JBI scoping reviews," 2015, https://nursing.lsuhsc.edu/JBI/docs/ ReviewersManuals/Scoping-.pdf.

[21] R. DerSimonian and N. Laird, "Meta-analysis in clinical trials," Controlled Clinical Trials, vol. 7, no. 3, pp. 177-188, 1986.

[22] M. Getachew, Assessment of postpartum contraceptive adoption and associated factors in Butajira health and demographic surveillance site (HDSS), in Southern Ethiopia, Addis Abeba Universty, 2015, http://etd.aau.edu.et/handle/123456789/ 11952.

[23] L. P. Zzimbe, "Determinants of postpartum contraceptive use among women aged 15-49 in Uganda," 2015, https://paa2015 .princeton.edu/papers/150278.

[24] M. E. Palamuleni, Use and determinants of postpartum contraception among women in Malawi, Population Training and Research Unit, North-West University, 2015.

[25] Y. Abera, Z. B. Mengesha, and G. A. Tessema, "Postpartum contraceptive use in Gondar town," Northwest Ethiopia: a community based cross-sectional study. BMC Womens Health, vol. 15, p. 19, 2015.

[26] T. H. Abraha, A. S. Teferra, and A. A. Gelagay, "Postpartum modern contraceptive use in northern Ethiopia: prevalence and associated factors - methodological issue in this crosssectional study," Epidemiol Health, vol. 39, article e2017019r, 2017.

[27] W. Ashebir and T. Tadesse, "Associated factors of postpartum modern contraceptive use in Burie district, Amhara region, Ethiopia," Journal of Pregnancy, vol. 2020, Article ID 6174504, 9 pages, 2020.

[28] S. S. Belda, S. G. Niguse, and K. A. Kuti, "Contraceptive use and associated factors among women in the extended postpartum period in Dello Mena district, Bale zone, Ethiopia: a crosssectional study," International Journal of Research Studies in Medical and Health Sciences, vol. 4, no. 12, pp. 1-14, 2019.

[29] G. A. Belete, A. A. Getu, and G. B. Gela, "Utilization and associated factors of modern contraceptives during postpartum period among women who gave birth in the last 12 months," in injibara town Awi Zone, North-West Ethiopia, 2019.

[30] M. Berta, A. Feleke, T. Abate, T. Worku, and T. Gebrecherkos, "Utilization and associated factors of modern contraceptives during extended postpartum period among women who gave birth in the last 12 months in Gondar town, Northwest Ethiopia," Ethiopian Journal of Health Sciences, vol. 28, no. 2, pp. 207-216, 2018.

[31] C. Bwazi, A. Maluwa, A. Chimwaza, and M. Pindani, "Utilization of postpartum family planning services between six and twelve months of delivery at Ntchisi district hospital, Malawi," Health, vol. 6, no. 14, pp. 1724-1737, 2014.

[32] L. Dulli, M. Green, and K. Katz, Increasing access to postpartum family planning in Madagascar: immunization services as an entry point to family planning for postpartum women, Family Health International, Durham (NC), 2010, https:// www.researchgate.net/publication/273692969.

[33] A. Gebremariam and H. Gebremariam, "Contraceptive use among lactating women in Ganta-Afeshum District, Eastern 
Tigray, Northern Ethiopia, 2015: a cross sectional study," BMC Pregnancy and Childbirth, vol. 17, no. 1, p. 421, 2017.

[34] W. Gizaw, "Extended postpartum modern contraceptive utilization and associated factors among women in Gozamen district, East Gojam Zone, northwest Ethiopia, 2014," Insights in Reproductive Medicine, vol. 1, no. 2, p. 8, 2017.

[35] D. N. Jaleta, H. Y. Yeshita, and K. S. Tamirat, “Timely initiation of postpartum contraceptive and associated factors among women of extended postpartum period in Pawe district, northwest Ethiopia," Internal Medicine, vol. 1, 2019.

[36] V. K. Kaydor, I. A. Adeoye, T. A. Olowolafe, and A. O. Adekunle, "Barriers to acceptance of post-partum family planning among women in Montserrado county, Liberia," Nigerian Postgraduate Medical Journal, vol. 25, no. 3, pp. 143-148, 2018.

[37] S. C. Keogh, M. Urassa, Y. Kumogola, S. Kalongoji, D. Kimaro, and B. Zaba, "Postpartum contraception in northern Tanzania: patterns of use, relationship to antenatal intentions, and impact of antenatal counseling," Studies in Family Planning, vol. 46, no. 4, pp. 405-422, 2015.

[38] Z. B. Mengesha, A. G. Worku, and S. A. Feleke, "Contraceptive adoption in the extended postpartum period is low in Northwest Ethiopia," BMC Pregnancy and Childbirth, vol. 15, no. 1, p. 160, 2015.

[39] A. Nigussie, D. Girma, and G. Tura, "Postpartum family planning utilization and associated factors among women who gave birth in the past 12 months, Kebribeyah town, Somali region, Eastern Ethiopia," Journal of Women's Health Care, vol. 5, no. 340, 2016.

[40] G. Rutaremwa, A. Kabagenyi, S. O. Wandera, T. Jhamba, E. Akiror, and H. L. Nviiri, "Predictors of modern contraceptive use during the postpartum period among women in Uganda: a population-based cross sectional study," BMC Public Health, vol. 15, no. 1, p. 262, 2015.

[41] M. Tegegn, M. Arefaynie, and T. Y. Tiruye, "Unmet need for modern contraceptives and associated factors among women in the extended postpartum period in Dessie town, Ethiopia," Contraception and reproductive medicine, vol. 2, no. 1, p. 21, 2017.

[42] P. N. Zivich, B. Kawende, B. Lapika, F. Behets, and M. Yotebieng, "Effect of family planning counseling after delivery on contraceptive use at 24 weeks postpartum in Kinshasa, Democratic Republic of Congo," Maternal and Child Health Journal, vol. 23, no. 4, pp. 530-537, 2019.

[43] H. A. Asires, S. Negash, and Y. Getiye, "Assessment of contraceptive need and practice of women in the extended postpartum period in Addis Ababa, Ethiopia [8G]," Obstetrics \& Gynecology, vol. 131, pp. 76-77S, 2018.

[44] A. Y. Gebremedhin, Y. Kebede, A. A. Gelagay, and Y. A. Habitu, "Family planning use and its associated factors among women in the extended postpartum period in Addis Ababa, Ethiopia," Contraception and Reproductive Medicine, vol. 3, no. 1, 2018.

[45] N. G. Gejo, A. A. Anshebo, and L. H. Dinsa, "Postpartum modern contraceptive use and associated factors in Hossana town," PLoS One, vol. 14, no. 5, article e0217167, 2019.

[46] F. Hounkponou, Y. Glèlè Ahanhanzo, C. O. A. Biaou et al., "Postpartum contraceptive use in Parakou (a city in Northern Benin) In 2018: a community based cross-sectional study," Open Access Journal of Contraception, vol. 10, pp. 19-26, 2019.
[47] K. Samuel, Factors associated with uptake of long acting reversible contraceptives methods among postpartum women at Juba teaching hospital: a cross sectional study, University of Nairobi, 2016.

[48] E. B. Taye, D. G. Mekonen, and T. Z. Debele, "Prevalence of post partum modern family planning utilization and associated factors among postpartum mothers in Debre Tabor town, North West Ethiopia, 2018," BMC Research Notes, vol. 12, no. 1, p. $430,2019$.

[49] Z. Tedla, Postpartum contraceptive use and associated factors among women of reproductive age group within the first 12 months of delivery in public health facilities of Addis Ababa, Ethiopia, Addis Ababa University, 2017.

[50] T. H. Abraha, B. B. Gebrezgiabher, B. G. Aregawi, D. S. Belay, L. T. Tikue, and G. M. Welay, "Predictors of postpartum contraceptive use in rural Tigray region, northern Ethiopia: a multilevel analysis," BMC Public Health, vol. 18, no. 1, p. 1017, 2018.

[51] B. S. Camara, A. Delamou, S. Sidibé et al., "Impact of an antenatal counseling on use of modern family planning methods in the postpartum in rural Guinea," African Journal of Reproductive Health, vol. 22, no. 4, pp. 16-25, 2018.

[52] A. N. Dasgupta, B. Zaba, and A. C. Crampin, "Postpartum uptake of contraception in rural northern Malawi: a prospective study," Contraception, vol. 94, no. 5, pp. 499-504, 2016.

[53] K. M. Sileo, R. K. Wanyenze, H. Lule, and S. M. Kiene, "Determinants of family planning service uptake and use of contraceptives among postpartum women in rural Uganda," International Journal of Public Health, vol. 60, no. 8, pp. 987-997, 2015.

[54] P. K. Rubee Dev, M. Feder, J. A. Unger, N. F. Woods, and A. L. Drake, "A systematic review and meta-analysis of postpartum contraceptive use among women in low- and middle-income countries," Reproductive Health, vol. 16, p. 154, 2019.

[55] M. Borda and W. Winfrey, Postpartum fertility and contraception: an analysis of findings from 17 countries, United States Agency for International Development (USAID), 2010.

[56] K. Sarvamangala and A. Taranum, "Menstrual pattern, sexual behavior and contraceptive use among postpartum women in a tertiary care hospital in Davangere," Medica Innovatica, vol. 2, no. 2, 2013.

[57] K. K. Singh, S. Verma, and S. Tanti, "Contraceptive use among postpartum women in India," Asian Population Studies, vol. 10, no. 1, pp. 23-39, 2014.

[58] S. Bibi, A. Shoukat, P. Maroof, and S. Mushraf, "Postpartum contraception utilization and its impact on inter pregnancy interval among mothers accessing maternity services in the public sector hospital of Hyderabad Sindh," Pakistan Journal of Medical Sciences, vol. 35, no. 6, pp. 1482-1487, 2019.

[59] K. B. Smith, "Is postpartum contraceptive advice given antenatally of value?," Contraception, vol. 65, pp. 237-243, 2002. 